\title{
Étude de l'ennui et du libertinage dans la littérature française du XVIIIe siècle : le libertinage est-il un bon remède à l'ennui ?
}

\author{
Raoudha Kallel, Dalhousie University
}

J'imagine que l'ennui d'une habitude où le cœur languit est la seule chose qui détermine une femme vers l'inconstance : elle ne voit plus dans un amant ces désirs tumultueux $[\ldots]^{1}$.

Il suffit de lire ce texte pour comprendre les raisons qui poussent une femme au libertinage. Selon Crébillon Fils, «l'ennui d'une habitude» est le plus grand coupable. Le raisonnement de Crébillon Fils est clair : sous l'effet de l'habitude, qui est une des conséquences fâcheuses des relations durables, l'ennui s'installe ; il transforme tout en froideur en entraînant non seulement l'affaissement de l'amour («le cœur languit») mais aussi la perte des désirs tumultueux. Aux yeux de celle qui s'ennuie, il existe un seul remède à l'ennui, un seul moyen de sortir de cette fadeur des sens et des sentiments : un nouvel amant et, par conséquent, une nouvelle liaison. D'une façon générale, le lien entre l'ennui et le libertinage n'est pas ambigu ; plusieurs textes, comme nous le verrons, en témoignent. Néanmoins, vu qu'aucune étude n’y a été exclusivement consacrée, une analyse approfondie des caractéristiques, des aspects et des enjeux de ce rapport ne serait pas inutile. C'est ce que nous nous proposons de faire dans cette étude dont l'objet est d'évaluer l'efficacité du libertinage en tant que remède à l'ennui. Notre étude se divise en deux parties : nous étudierons, en premier lieu et à titre d'introduction, le lien entre l'ennui et le libertinage chez des personnages féminins dans le but de montrer la place qu'occupe la libertine dans la littérature des Lumières, un sujet qui a été peu traité par la critique. Dans la deuxième partie, nous élargirons notre champ d'analyse et en nous basant sur des modèles aussi bien féminins que masculins, nous examinerons l'ennui dans son rapport avec les 
thèmes libertins suivants : la valorisation du plaisir, l'éloge de l'inconstance, l'importance du corps et de la sensualité physique, la conception négative de l'amour et le culte de soi.

Il est onze heures du matin. Un rayon de soleil, qui transperce le volet, vient éclairer la chambre à coucher. La marquise se réveille, relève le rideau, caresse son bichon et sonne. La femme de chambre accourt pour servir Madame. Petit-déjeuner, toilette, coiffure, habillage : alors que la servante s'occupe de l'apparence de sa maîtresse, celle-ci s'absorbe dans ses réflexions. Pour que sa journée soit couronnée de succès, elle n'a pas de temps à perdre ; il lui faut choisir ses victimes et préparer ses conquêtes : des idées machiavéliques qu'elle mettra plus tard en action.

La toilette finie, les cheveux nattés, le corps serré dans une robe de la dernière mode ; le grand moment du lever du rideau est arrivé et voici la marquise qui s'apprête à mener le jeu. Avec un air qui affiche sa supériorité, elle reçoit son public. C'est un chevalier, ou un marquis, l'amant du jour dont elle se sert pour satisfaire ses désirs et faire triompher ses vengeances. C'est un comte qu'elle considère, aux besoins de la scène, tantôt comme complice et tantôt comme adversaire. C'est une cousine qui vient lui raconter, innocemment, ses soucis concernant sa fille, sortie du couvent. Et au milieu de ce cercle de familiers, les yeux saillants de malice et de coquetterie, elle trompe et séduit, domine et influence, se venge et compatit. Véritable actrice, elle joue parfois la bonne amie et la tendre tante, parfois la méchante femme et la capricieuse amante. Experte dans l'art de feindre et de tromper, elle met tout à son service pour arriver à ses fins : personnes, écriture, objets, mots, lieux, fiction, etc. Bref, rien ne lui échappe, tout est calculé : à chaque scène, un scénario ; à chaque acte, un masque ; à chaque correspondant, un style. Cet esprit du mal à l'état pur, c'est la marquise de Merteuil qui l'incarne lorsque Laclos en fait le portrait dans Les Liaisons dangereuses. 
Si, au goût de Mme Riccoboni, Laclos pousse trop loin la théorie du libertinage féminin², il n'en est cependant pas l'inventeur. Nombreux sont, en effet, les romanciers qui mettent en scène des libertines scélérates. Dans Les Égarements du cœur et de l'esprit, Crébillon Fils présente le personnage de Mme de Lursay qui, experte dans l'art de séduire au même titre que Versac, enseigne au jeune Meilcour des cours de libertinage. Le narrateur la décrit ainsi :

[Mme de Lursay] avait l'esprit vif, mais sans étourderie, prudent, même dissimulé. [...] Elle avait étudié avec soin son sexe et le nôtre, et connaissait tous les ressorts qui les font agir. Patiente dans ses vengeances, comme dans ses plaisirs, elle savait les attendre du temps, lorsque le moment ne les lui fournissait pas. (Les Égarements du cour et de l'esprit, 15)

Quelques années plus tard, Dorat vient augmenter le nombre de ces libertines lorsqu'il crée le personnage de Mme d'Ercy dans Les Sacrifices de l'Amour. Comme ses consœurs, elle est tout à fait capable de toutes les perfidies : quête des plaisirs voluptueux, goût de la vengeance, volonté de domination. C'est dans ces termes que Versenai, qui lui doit beaucoup d'ailleurs, peint les traits de son caractère :

Léger, superficiel, altier. Sa tête la trompe sur les mouvements de son cœur : Dieu sait ce qui résulte de ce faux calcul. [...] Elle est jalouse avec hauteur, exigeante sans tendresse, capricieuse à un excès que je peindrais mal, et le caprice est presque toujours chez les femmes en proportion de leur froideur. (Les Sacrifices de l'amour, 20)

Mais le portrait de la libertine la plus accomplie vient certainement de la plume de Vivant Denon, l'auteur de Point de Lendemain dont l'héroïne réussit à tromper à la fois son mari, son amant en titre et un jeune innocent qui lui sert d'amant d'un soir, sans pour autant perdre ni sa dignité ni sa réputation. Dans un décor luxueux qui met en relief la théâtralité de cette véritable 
mise en scène, les gestes érotiques se succèdent et s'accélèrent. Dans son grand égoïsme immoral, la maîtresse réduit son amant à une sorte de marionnette dont elle se sert pour sa propre satisfaction charnelle.

Le libertinage est donc loin d'être uniquement une vocation masculine. Bien au contraire, les femmes ne sont pas moins libertines que les hommes. Charme, esprit, mais aussi perversité et rouerie: c'est certainement l'image du «démon femelle »(Versini 105), pour reprendre l'expression de Laurent Versini, qui se reflète dans les romans du XVIIIe siècle. En ce « siècle du libertinage »(Cazenobe 7), il n'est pas surprenant que la femme occupe une place importante dans la comédie des libertins. Michel Delon reconnaît ce fait :

La libertine [...] brave la condamnation morale et jouit des applaudissements de ses semblables. [Elle] prend le relais du roué masculin et peut confondre éducation intellectuelle et séduction physique des jeunes gens qui entrent dans le monde. (Delon, 34)

Ainsi donc, se plaît-elle à semer «les fleurs du mal » autour d'elle. Nul doute que derrière «les fleurs du mal » se cache l'ennui, cet ennui qui évoque le passé et rappelle l'avenir : il se nourrit, d'une part, de l'ennui pascalien puisqu'il est lié au vide de l'existence ; il se nourrit, d'autre part, de l'ennui baudelairien en faisant prévaloir toutes les formes du dégoût (le dégoût de soi, de la vie, des autres... le dégoût de tout).

Si le libertinage s'inscrit dans le cadre d'une mode caractéristique de l'aristocratie, il traduit aussi une attitude de réaction contre l'ennui. La crainte de l'ennui entraîne en effet le désir du libertinage. C'est pour repousser l'ennui que la libertine se réfugie dans le libertinage. Cette doctrine, qui privilégie avant tout la volupté dans toutes ses formes, lui paraît prometteuse dans sa lutte contre l'ennui. Plus concrètement, puisque l'ennui est une réalité faite de platitude 
et de néant, il faut l'attaquer sous forme de transcendance du plaisir extrême. De la conscience de l'ennui naît fondamentalement la conscience voluptueuse. C'est sous cet angle que Duclos analyse le comportement libertin de Madame de Persigny :

[...] c'était alors que l'imagination de Madame de Persigny travaillait, que les messages couraient, et qu'il était indispensablement nécessaire de trouver de quoi remplir un intervalle qui se trouvait vide. La crainte de l'ennui était un ennemi pour elle : c'était lorsqu'il fallait remplacer une partie qu'elle devenait caressante ; son esprit était insinuant, et c'est avec ce caractère que la femme la plus extravagante fait approuver et partager aux hommes toutes les folies (Les Confessions du Comte de ${ }^{* * *}, 225$ ).

En fait, puisque le libertinage est une école qui dicte les mêmes principes à ses élèves, le comportement libertin de la femme n'est pas du tout différent de celui de l'homme. Les axes de similarité se recoupent sur tous les plans et les mêmes égarements du cœur et de l'esprit caractérisent ce qu'on appelle le libertinage mondain au XVIIIe siècle. Le concept de libertinage féminin n'est ni original ni spécifique. On l'introduit dans le cadre de notre étude comme une justification supplémentaire du rapport entre l'ennui et le libertinage. Dans la suite de notre analyse de l'ennui et du libertinage, le discours masculin accompagnera son homologue féminin.

Tout bien considéré, le lien entre l'ennui et le libertinage est loin d'être imperceptible. Dans la littérature du XVIIIe siècle, ils apparaissent, en effet, comme un couple indissociable. Beaumarchais le remarque d'ailleurs dans la bouche de Bartholo qui déclare : «mon maitre est libertin par ennui »(Le Mariage de Figaro, Acte I, scène 4). On retrouve une affirmation semblable sous la plume de Dorat qui définit le libertin comme « un homme [qui] promène dans la société son ennui inquiet. »(Les Malheurs de l'inconstance, 105) Crébillon Fils, conscient de 
cette interdépendance entre ennui et libertinage, fait aussi du libertin un misérable «ennuyé »; son héros Meilcour s'adonne corps et âme au commerce des femmes, justement par ennui :

Au milieu du tumulte et de l'éclat qui m'environnaient sans cesse, je sentis que tout manquait à mon cœur : je désirais une félicité dont je n'avais pas une idée distincte ; je fus quelque temps sans comprendre la sorte de volupté qui m’était nécessaire. Je voulais m'étourdir en vain sur l'ennui intérieur dont je me sentais accablé ; le commerce des femmes pouvait seul le dissiper (Les Égarements du cour et de l'esprit, 10).

Tandis que l'ennui est une désolation, le libertinage apparaît comme une consolation. L'humiliation de l'ennui fait la grandeur du libertinage. Et ces désœuvrés, ces victimes de l'ennui, se transforment volontairement en libertins cyniques pour qui le plaisir devient un dieu. C'est à travers cette quête des plaisirs des sens ${ }^{3}$ que les libertins recherchent une victoire sur l'ennui et font du plaisir le fondement d'une morale. Dans son traité sur L'Art d'aimer et poésies diverses, Gentil-Bernard prône une morale hédoniste qui idéalise le plaisir et rabaisse l'ennui :

L'homme [...] sans âme et sans force,

D'aucun penchant ne connaissant l'amorce ;

Séché d'ennui, de langueur consumé ;

Obscur, rampant, vivant inanimé ;

Réduit, sans voir, sans jouir, sans connaître ;

Au froid plaisir de végéter et d'être ;

S'il eut alors des succès éclatants ;

Si l'art d'aimer fut le même en tout temps, L'art de jouir augmenta d'âge en âge. (L'Art d'aimer et poésies diverses, 34) 
De plus, c'est dans ces vers tirés de ses Poésies érotiques que Parny exprime sa conception du plaisir, conception opposée à celle de l'ennui :

Va, crois-moi, le plaisir est toujours légitime ;

L'amour est un devoir, l'ennui seul est un crime. (Poésies Érotiques, 26)

Parny ne s'en tient pas à décrire le plaisir comme légitime. Aux Infidèles, il présente ses hommages :

Pour moi, je vous dis grand merci

Vous seuls de ce triste monde

Avez l'art d'égayer l'ennui ;

Vous seuls variez la scène

De nos goûts et de nos erreurs ;

Vous piquez au jeu les acteurs ;

Vous agacez les spectateurs

Que la nouveauté vous amène. (Poésies Érotiques, 50)

Écrivains et poètes enseignent donc l'art d'égayer l'ennui. Leur leçon est simple : il ne faut songer qu'aux jouissances de la vie. C'est dans le cadre d'une didactique du plaisir que les libertins, loin d'être coupables, passent pour des modèles. Leur conduite devient un exemple ; leur jeu devient un défi ; leur frivolité devient une arme triomphante qui vise à combattre l'ennui. Dans ce contexte, l'ennui de l'uniformité s'oppose clairement au plaisir de la nouveauté. Alors que l'amour honnête est source d'ennui, la multiplicité des liaisons semble être au contraire source de plaisirs infinis. La passion est lieu de désespoir, de dégoût et d'emprisonnement ; seule l'inconstance est plaisante. Véritable ressort de la volupté, elle dispose à l'espoir et à l'optimisme, car elle libère l'âme et lui ouvre «la route des plaisirs » (Angola, 100). 
L'inconstance cesse d'être un crime ; elle devient plutôt une pratique normale, courante, au sein de la bonne compagnie. Comme le plaisir, elle devient le fondement d'une nouvelle morale. Ainsi, la littérature des Lumières renferme-t-elle de nombreux réquisitoires contre la constance et l'uniformité dans les liaisons. En voici quelques exemples :

Tableau 1 : Réquisitoires contre la constance et l'uniformité dans les liaisons

\begin{tabular}{|c|c|c|}
\hline Auteur & Citations & Références \\
\hline Marmontel & $\begin{array}{l}\text { je vois que le plus grand des maux } \\
\text { qui affligent l'humanité, c'est } \\
\text { l'ennui : or l'ennui vient de } \\
\text { l'égalité dans le caractère; de la } \\
\text { constance dans la liaison; de la } \\
\text { solidité dans les goûts, de la } \\
\text { monotonie enfin qui endort le } \\
\text { plaisir. }\end{array}$ & Contes Moraux, T. 1, 192. \\
\hline Duclos & $\begin{array}{l}\text { Les sentiments de la marquise ne } \\
\text { me touchaient plus. Je ne sentais } \\
\text { que l'ennui et le dégoût d'un } \\
\text { plaisir uniforme. }\end{array}$ & $\begin{array}{l}\text { Les Confessions du comte } \\
\text { de ***, 1741, in Romans } \\
\text { libertins du XVIIIe siècle, } \\
\text { 186. }\end{array}$ \\
\hline Crébillon Fils & $\begin{array}{l}\text { Elle vous demande ce que vous } \\
\text { pensez de la constance; vous } \\
\text { répondez ingénument qu'il n'est } \\
\text { rien de plus ennuyeux. }\end{array}$ & $\begin{array}{l}\text { Lettres de la Marquise de } \\
M^{* * *} \text { au comte de } R^{* * *}, \\
126 .\end{array}$ \\
\hline
\end{tabular}

Procurant «l'agrément de la variété » (De Pouilly, 17), l'inconstance apparaît comme une force d'enchantement assez suffisante en vue de combattre l'ennui. Puisqu'elle tient aussi de la nature, comme le précise Bernard Lamy, il n'est pas surprenant qu'elle soit le principe capital qui caractérise toute psychologie libertine. En fait, ce principe n'est pas seulement visible chez le roué, il influe aussi sur le comportement de la petite-maîtresse. "On se convient, on s'ennuie, et on se quitte ${ }^{4}$ (Contes Moraux, T.2, 16) : c'est en suivant cette logique que la libertine se trouve entraînée par la nécessité des changements auxquels engage l'inconstance elle-même. Qu'on en juge par ce portrait que Marmontel fait d'Artenice : 
Artenice était une de ces femmes pour qui l'amour est un arrangement de société, qui s'offensent d'un long respect, qui s'ennuient d'un amour constant, et qui comptent assez sur la probité des hommes, pour s'y livrer sans réserve, et les quitter sans ménagement. (Contes Moraux, T. 2, 11)

Avec la figure de la femme cavalière et libertine, le discours féminin dévoile l'aspiration idéale qui préside à toutes les conquêtes et à tous les désirs des libertines. Un tableau vivant se dessine alors en faveur de l'inconstance. Dans les Bijoux indiscrets de Diderot, Fanni critique la manière dont « on aime à présent » et expose ses idées sur l'inconstance :

En prendre à son aise; tenir tant qu'on s'amuse ; quitter dès qu'on s'ennuie, ou que la fantaisie parle pour un autre. L’inconstance offre une variété de plaisirs inconnus à vous autres transis. (Les Bijoux indiscrets, 168)

De la théorie à la pratique : dans une mise en scène où la sensualité excite le désir, Mademoiselle de $\mathrm{D}^{* * *}$ décrit la vivacité de ses sensations lorsqu'elle commet, dans l'obscurité de la nuit et sans le savoir, l'acte d'inconstance. L'extrait suivant tiré d'Antipaméla offre un véritable spectacle de volupté où l'éloquence vient au service de l'héroïne pour plaider la cause de l'inconstance :

Je n'avais jamais ressenti une volupté si piquante : le chevalier lui-même semblait avoir redoublé de vivacité. Ciel ! Disais-je en moi-même, que les plaisirs dérobés sont doux ! [...] Le jour commençait à peine à paraître. Mon amant dormait encore ; j'écartai un des rideaux pour le contempler ; mais, quel prodige! Je reculai d'effroi, en reconnaissant Keil, c'était lui qui m'avait écrit sous le nom du Chevalier ; c'était lui [...] à qui j'avais prodigué les plus tendres caresses, en croyant ne le faire que pour un amant favorisé. [...] J'allais éclater, lorsqu'une réflexion m'arrêta. J'étais outrée du tour qu'il m'avait joué ; 
mais, le dirais-je? Le ressouvenir de la nuit me demandait grâce pour lui. [...] Le plaisir a diverses faces? Je ne l'avais jamais envisagé sous celle qu'il m'offrait. La nouveauté de l'objet me séduisit ; je suivis la pente qu'elle me présentait ; mes sens en reçurent l'image enchanteresse [...]. La sensualité prévalut sur l'amour de sentiment. (Antipaméla, 97-101)

«La sensualité prévalut sur l'amour de sentiment »: cette conclusion est sans doute révélatrice de l'esprit libertin selon lequel les mouvements du corps triomphent de ceux du cœur. C'est le corps qui dicte, pour ainsi dire, les véritables lois du désir. Quant au cœur, il éveille simplement les sentiments qui créent, semble-t-il, des illusions pénibles. Le corps procure, en effet, des plaisirs réels, vécus, authentiques ; le cœur est susceptible de sentimentalisme qui trouve sa force dans les fantasmes du rêve et de l'imagination. À l'encontre du roman sentimental dont Rousseau et Madame Riccoboni assurent le succès, le roman libertin fait la représentation de la mécanique des «sentiments du corps $»^{5}$. Il s'agit d'une conception matérialiste des sentiments. De ce point de vue, le sentiment est loin d'être le fruit de la vie affective ; il n'est acceptable que dans la mesure où il fait naître une sensibilité instinctuelle, corporelle, «une sensibilité physique » (De l'esprit, 2), pour reprendre l'expression de Helvétius. Le vrai sentiment est «celui que le plaisir fait naître ${ }^{6}$ (Les bijoux indiscrets, 229), affirme Diderot dans les Bijoux indiscrets. À proprement parler, l'idée du sentiment n'existe guère en dehors du corps. Cette action mécanique des sentiments est évidente aussi chez Fougeret de Monbron. ${ }^{7}$

Chez le libertin, alors que l'art d'aimer n'est qu'un leurre, l'art de jouir est une réalité thérapeutique qui ferme les portes à l'ennui. C'est à partir de la conscience libertine que l'on peut comprendre la conception, certainement amorale, de l'amour ${ }^{8}$ chez les libertins. Il s'agit sans doute d'un amour masqué et malhonnête. Incapables d'aimer de peur de perdre leur 
autonomie affective, les libertins se servent en effet des sentiments pour tromper autrui et dominer sur autrui. Dans ce contexte, les liens qu'ils nouent avec leurs partenaires ne ressemblent pas du tout aux liens habituels de l'amour réciproque. Il s'agit plutôt d'une relation de maîtres (ou de maîtresses) envers leurs esclaves dont il faut se servir pour affirmer leur propre supériorité et pouvoir dominer l'autre. En fait, dans le cadre de leur volonté de supériorité, les libertins n'ont pas besoin de partenaires ; ils ont plutôt besoin de victimes auprès desquelles ils ne cherchent qu'une distraction. Ils ne recherchent pas l'amour, mais plutôt des aventures qu'ils préparent minutieusement et lucidement sans que la moindre sensibilité ne vienne déranger leurs entreprises. Et pas n'importe quelles entreprises, mais seulement celles qui présentent des difficultés, les obstacles les plus insurmontables. La difficulté prend ainsi plusieurs valeurs : si elle « est un divertissement, c'est-à-dire une façon d'oublier l'ennui existentiel » ${ }^{9}$ (Nordon, 91), elle traduit aussi une volonté de dépassement et un moyen de prouver sa toute-puissance à l'égard des autres tout en exaltant sa prouesse. C'est la raison pour laquelle Valmont accorde une importance particulière au projet de séduire la Présidente de Tourvel :

Dépositaire de tous les secrets de mon cœur, je vais vous confier le plus grand projet que j’aie jamais formé... Vous connaissez la Présidente de Tourvel, sa dévotion, son amour conjugal, ses principes austères. Voilà ce que j'attaque ; voilà l'ennemi digne de moi ; voilà le but où je prétends atteindre. (Les Liaisons dangereuses, 41)

L'amour n'est, par conséquent, ni le but ni la préoccupation des libertins, il est simplement un moyen de séduction, une tactique parmi d'autres pour s'assurer le succès des conquêtes. Sur ce sujet, l'exemple de la Marquise de Merteuil est convaincant : se voulant experte dans l'étude du cœur humain, elle se sert des manifestations du sentiment pour conduire l'objet de sa conquête dans le piège qu'elle lui a tendu. Ayant appris, dès sa jeunesse, à « régler [...] les divers 
mouvements de [sa] figure »(Les Liaisons dangereuses, 200), elle joue la «femme sensible» (Les Liaisons dangereuses, 204) et n'hésite jamais à pleurer. Ainsi écrit-elle à Valmont : «Mais voulant frapper le coup décisif, j'appelai les larmes à mon secours » (Les Liaisons dangereuses, 218). À une autre occasion, lorsqu'elle s'ennuie de son aventure avec Belleroche, elle décide de « lui montrer plus d'amour, pour en venir à bout plus facilement [...]. [Elle] le surcharger[a] à tel point d'amour et de caresses »(Les Liaisons dangereuses, 295). Les différentes manifestations de la sensibilité (larmes, caresses, etc.) deviennent, par conséquent, une arme que les libertins utilisent pour feindre le sentimentalisme et manipuler leurs victimes : une compétence qui confirme certainement la primauté de l'intelligence sur le cœur. Cet extrait d'une lettre de la Marquise de Merteuil met en lumière cette ambivalence :

Une fois fixée sur ces trois objets, le dernier seul présentait quelques difficultés dans son exécution ; j’espérai les vaincre et j’en méditai les moyens. Je commençai à m’ennuyer de mes plaisirs rustiques, trop peu variés pour ma tête active ; je sentais un besoin de coquetterie qui me raccommoda avec l'amour ; non pour le ressentir à la vérité ; mais pour l'inspirer et le feindre. (Les Liaisons dangereuses, 96)

Or pour pouvoir garder la suprématie de l'esprit et l'autonomie de la volonté, il est indispensable de rejeter tout lien affectif. En effet, toute marque d'amour représente un danger parce qu'elle entrave le bon fonctionnement de la pensée : «votre cœur abuse votre esprit »(Les Liaisons dangereuses, 346), écrit la Marquise à Valmont. Parce qu'il égare l'intelligence, paralyse la volonté, aliène la lucidité et entraîne le délire, l'amour représente sans équivoque une vraie menace à laquelle il faut échapper pour ne pas tomber dans l'abrutissement de l'être dont témoigne sans doute la passion de Madame de Tourvel. De cette conscience du danger naît un mépris pour l'amour et pour tout engagement sentimental. Aux yeux du libertins, l'amour est 
indigne ; c'est un mal, affirme Mme de Merteuil : «Je dis l'amour; car vous êtes amoureux. Vous parler autrement, ce serait vous cacher votre mal »(Les Liaisons dangereuses, 52). Les libertins le considèrent en fait comme un lieu d'échec, de faiblesse et d'avilissement. L'amour d'autrui est ennuyeux, «Pusillanime »(Les Liaisons dangereuses, 320), il ne mène à rien sinon à l'esclavage et à la contrainte. C'est l'amour de soi qu'il faut, par contre, savoir cultiver. Le vrai amour est l'amour de soi. Le vrai triomphe est aussi le triomphe de soi qui conduit véritablement au sommet de la gloire. À cet égard, le libertinage, s'opposant au conformisme traditionnel, trouve son essence non pas dans l'essor de la sensibilité, mais plutôt dans le culte d'un individualisme absolu. Dans Laclos: Connaissance des Lettres, René Pomeau explique l'importance de cette éthique du moi chez les libertins :

Le libertin pratique le culte de son moi. Ses prouesses : autant d'exercices où s'éprouve et se confirme le sentiment de son excellence. Champion solitaire, pour qu'il déploie ses talents, il est utile que soient coupés autour de lui les liens qui retiennent l'homme ordinaire. Aussi ce désert familial [...] est exigé aussi par la logique du personnage. [...] Point de frère [...], ni d'oncle. (112)

Pomeau se sert de l'expression « désert familial » pour faire ressortir la dimension typiquement existentielle du libertinage. Si le libertin choisit le désert, ce n'est certainement pas par une sorte de lâche misanthropie ; bien au contraire, le libertin ne peut vivre sans autrui. Cependant, lorsqu'il est loin de son public, il n'a pas de temps à perdre avec la famille. Il lui faut plutôt mettre à l'épreuve ses prouesses et s'assurer de l'excellence de son moi pour qu'il puisse dominer l'autre et conquérir le monde. Or, dans cet univers où le libertin est maître de soi, y aurait-il vraiment un remède à l'ennui ? 
Ernest Sturm parle du «crépuscule du libertinage» (107) ; Philippe Laroch décrit «le déclin du libertinage » et évoque l'idée de «la fin d'un mythe ${ }^{10}$; Colette Cazenobe conclut à un «échec du libertinage » (444). Autant de réflexions qui témoignent de la dégradation du libertinage comme système social. Cette dégradation est, à juste titre, la preuve de l'impuissance du libertinage à apporter le bonheur ${ }^{11}$. La ronde perpétuelle des divertissements artificiels, l'absence de spontanéité dans ce jeu continuel de dissimulation, le mépris de l'autre et la fierté de soi : toutes ces raisons contribuent à rendre bientôt les plaisirs insipides et monotones. Le libertinage n'est, en fait, qu'une autre forme de monotonie. L'enchaînement des plaisirs devient avec le temps une habitude, une routine comme toute autre. À cet égard, le raisonnement par analogie de Rémy de Gourmont est intéressant :

L'ennui ! Mot terrible et justement redouté ! Que de remèdes [...] souvent plus ennuyeux encore que l'ennui même. Leur nom général est «plaisirs » [...]. Les plaisirs quoique abondants et communs, sont une recherche, et presque toujours vaine. Quand on réussit à opposer au géant Ennui l'armée des nains Plaisirs, le géant étouffe les nains en quelques gestes et reprend sa pose lassée. L'ennui, à vrai dire, est invincible. (211)

Les plaisirs sont donc de faux-remèdes. Même l'inconstance, à force de la répéter, perd de sa volupté et se transforme elle-même en uniformité ${ }^{12}$. Elle devient une sorte de paradis perdu. C'est dans ces termes que Mademoiselle de $\mathrm{D}^{* * *}$ explique les raisons de cet émoussement :

Tout nous frappe dans nos premières années : nous saisissons avec avidité les objets qui nous environnent, le sentiment qu'ils excitent en nous pénètre jusqu'à notre âme avec d'autant plus de facilité, qu'il ne rencontre aucun obstacle. Cette disposition à recevoir les impressions, s'émousse insensiblement par l'habitude où nous sommes de les recevoir (Antipaméla, 1-2). 
Ainsi, les vrais visages du libertinage se dévoilent: non seulement se révèle-t-il incapable de combattre l'ennui, il conduit aussi à la triste réalité de l'ennui. À vrai dire, l'ennui est consubstantiel au libertinage. De toute évidence, le « théâtre des méchants ${ }^{13}$ est aussi un théâtre de l'ennui. Dans leur perfectionnisme outré, les libertins confrontent partout l'uniformité et, par conséquent, l'ennui. Avides de désirs, ils se mettent à multiplier les liaisons. Or, le désenchantement persiste et chaque nouvelle liaison débouche sur une autre crise d'ennui. La conclusion est la même pour toute liaison : alors que le début enchante, la fin ennuie. Prônant la liberté sous toutes ses formes, les libertins se trouvent paradoxalement enfermés dans ce cercle vicieux où plaisirs et ennui s'alternent et se succèdent interminablement. Ce zigzag perpétuel finit par rompre l'équilibre entre les «amusements de l'âme » (Le Sopha, «préface », VI) et ceux du corps. Il en résulte la lassitude. Les libertins sont inévitablement condamnés à l'ennui des liaisons.

On peut donc parler de l'ennui du libertinage. Cette catégorisation de l'ennui met en lumière deux aspects qui semblent être, pour le moins, contradictoires. Alors que l'ennui est une maladie intérieure et morale qui s'empare de l'âme, le libertinage se définit comme un système social, voire une philosophie de vie qui privilégie l'excès de matérialité et tire sa force de l'univers extérieur, de l'entourage de l'individu. C'est en fait de cette incompatibilité entre le mal (l'ennui) et le remède (le libertinage) que naît un aspect important de cet ennui du libertinage. En effet, comme nous l'avons déjà analysé, c'est pour combattre l'«ennui intérieur » (Les Égarements du cœur et de l'esprit, 10) que les libertins se livrent sans gêne et sans scrupule au libertinage. Les plaisirs du jeu, les plaisirs de la séduction, les plaisirs de la vengeance, les plaisirs de la chair, les plaisirs du pouvoir : lieu de «félicité » (Les Égarements du cour et de l'esprit, 10), le libertinage se présente, en apparence, comme l'école du plaisir où le bonheur 
s'annonce en permanence. Accablés d'ennui et avides de joie de vivre, les libertins font du libertinage leur passion. Ils se mettent, par conséquent, à le cultiver avec art. L'art de tromper, l'art de séduire, l'art de conquérir: c'est en un mot l'art de faire le mal que les libertins pratiquent pour guérir leur malaise intérieur. D'ailleurs, c'est sous cet angle que Ronald C. Rosbottom analyse le comportement de Valmont et de la Marquise de Merteuil :

As libertines, they are by nature arch-enemies of boredom ; they plot and scheme because they are bored. In fact, the ethics of libertinism justify any attempt that seeks to alleviate ennui. [...] they are bored with any affair that lingers (Letter 113); they are bored with writing and copying letters (Letters 34 and 51); they are bored with others in society (Letter 110), and so forth. The entire novel, in fact, is a commentary on social boredom and the means employed to lessen its impact. Bored at the beginning of the novel, Merteuil and Valmont, in order to alleviate that boredom, form plots to manipulate others [...]. (16-17)

Mensonge, dissimulation, hypocrisie, chantage, vengeance, jeu, machiavélisme, conquêtes : les libertins s'engagent fidèlement à mettre en action l'anti-morale dont Versac énumère les principes dans Les égarements du cour et de l'esprit de Crébillon.

Tableau 2 : Principes libertins

\begin{tabular}{|l|c|}
\hline \multicolumn{1}{|c|}{ Principes libertins } & Références \\
\hline Vous devez apprendre à déguiser si parfaitement votre caractère. & 172 \\
\hline $\begin{array}{l}\text { Il faut encore que vous joigniez à l'art de tromper les autres, celui de les } \\
\text { pénétrer. }\end{array}$ & 172 \\
\hline $\begin{array}{l}\text { Il vaut souvent mieux donner mauvaise opinion de son esprit, que de } \\
\text { montrer tout ce qu'on en a. }\end{array}$ & 172 \\
\hline $\begin{array}{l}\text { Une chose encore extrêmement nécessaire, c'est de ne s'occuper jamais } \\
\text { que du soin de se faire valoir. }\end{array}$ & 173 \\
\hline $\begin{array}{l}\text { Il est plus sûr de subjuguer les autres, que de leur immoler sans cesse les } \\
\text { intérêts de notre amour propre. }\end{array}$ & $173-174$ \\
\hline $\begin{array}{l}\text { Être passionné sans sentiment, pleurer sans être attendri, tourmenter sans } \\
\text { être jaloux : voilà tous les rôles que vous devez jouer, voilà ce que vous }\end{array}$ & 176 \\
\hline
\end{tabular}


devez être.

Mais n'est-ce pas une entreprise infructueuse que de prétendre guérir une maladie morale par le moyen de l'immoralité ? Le moral ne se nourrirait pas de l'immoral. Le moral ne se réconcilierait pas avec l'immoral. L'incompatibilité entre le mal et le remède s'avère inévitable. La guerre des libertins contre l'ennui ne réussit pas et l'univers de l'immoralité dans lequel ils baignent accentue ce sentiment du vide intérieur dont ils sont victimes. La recherche excessive des plaisirs matériels et des dissipations aux dépens d'autrui, l'épanouissement du moi dans le mal finissent par faire naître le sentiment d'un vide moral. Il n'existe aucun fondement moral chez les libertins. Le mal leur tient lieu d'éthique. En fait, ils n'ont même pas d'âme, comme l'explique Jean Sgard :

Les libertins n'en ont pas [d'âme] : totalement socialisés, ils ont perdu leurs sentiments, leurs rêves, leur for intérieur. Ils n'en sont pas moins habités par une sorte de nostalgie, qui se traduit d'abord par l'ennui. (Le Sopha, « Préface », VI-VII)

Une nostalgie des valeurs morales? C'est, en tout cas, sous forme d'un grand vide moral que l'ennui des libertins s'affirme et se réaffirme. Un vide moral qui fait naître de tristes réflexions, comme celle qui est faite par Zulma : «Las de la vie errante que je menais, [...] je commençai à m'ennuyer de ma destinée » (Le Sopha, 79) ; et un peu plus tard il ajoute : «[...] las encore de mes courses, dégoûté du monde, sentant alors avec horreur à quel point il m'avait perverti, je n'étais pas fâché d'entendre morale, [...] je la regard[ai] comme une chose qui pouvait m'être salutaire $\gg(82)$.

Au vide moral vient s'ajouter le vide sentimental. En effet, si les libertins ressentent une nostalgie des valeurs morales, il ne sera pas étonnant qu'ils éprouvent aussi une nostalgie de l'amour. Comme nous l'avons déjà vu, les libertins ont une vision négative de l'amour. Fiers, 
libres, intelligents et indépendants, l'amour est leur plus grand ennemi. Il symbolise la soumission, entraîne le désordre total de l'être et nuit à la pensée. D'ailleurs, on ne manquerait pas d'exemples, dans la littérature libertine du XVIIIe siècle, de réquisitoires contre l'amour ${ }^{14}$.

Cependant, derrière ce refus de sentimentalité qui est associé aux principes libertins, se cache une certaine attirance envers l'amour. Le cour, qui a besoin de sentiments, y incite les libertins. Lorsque ces derniers refusent de s'y prendre, le cœur devient insatisfait. Cette secrète insatisfaction entraîne le vide du cœur. L'ennui des libertins se traduit par le vide du cœur. Le Chevalier, un des héros libertins de Dorat, en est conscient lorsqu'il s'exclame ainsi :

Qu'est-ce, hélas! Que la gloire, quand le cœur est vide, isolé par l'orgueil [...] ? L'ambition n'est que le dédommagement des êtres froids. N'ayant ni vertu qui les invitent à se recueillir, ni sentiments qui les y forcent, il leur faut des erreurs qui les jettent au-dehors [...]. (Les Sacrifices de l'amour, 52)

De cette étude, surgit une vérité concrète : qu'il soit l'incarnation du mal à l'état pur, le libertin est avant tout victime de l'ennui, d'un ennui qui subsiste partout au sein même des plaisirs. Il se jette au libertinage pour s'échapper à l'ennui. Paradoxalement, ce sont les principes mêmes du libertinage qui l'entraînent vers l'ennui, vers ce que nous avons identifié comme le vide du cœur et le vide moral. Enfin, si le libertin veut combattre son ennui, ne lui faut-il pas retourner à la morale ? Peut-être à une morale que Pascal, l'auteur des Pensées, définit comme «la misère de l'homme sans dieu »?

\section{Ouvrages cités}

Beaumarchais. Le Mariage de Figaro, Paris : Larousse, 1971.

Bernard, Pierre Joseph. L'Art d'aimer et poésies diverses. Paris : Lejay, 1775. 
Laroch, Philippe. Petits-maîtres et roués : évolution de la notion de libertinage dans le roman français du XVIII siècle. Québec : PU Laval, 1979.

Bernier, Marc André. Libertinage et figures du savoir : Rhétorique et roman libertin dans la France des Lumières (1734-1751). Québec : PU Laval, 2001.

Cazenobe, Colette. Le système du libertinage de Crébillon à Laclos. Studies on Voltaire and the Eighteenth Century, v. 282, Oxford: The Voltaire Foundation, 1991.

Crébillon Fils, Claude-Prosper Jolyot de. Les égarements du cœur et de l'esprit. Paris : Colin, 1961.

----. Le Sylphe. Paris : Librairie des Bibliophiles, 1873.

----. Le Sopha. Paris : Desjonquères, 1984.

Delon, Michel. P.-A. Choderlos de Laclos, Les Liaisons dangereuses. Première édition. Paris : PUF, 1986.

Denon, Vivant. Point de lendemain. Paris : Desjonquères, 1987.

De Pouilly, Louis-Jean Lévesque. «Théorie des sentiments agréables. Où l'on établit les principes de la morale. »Recueil de divers écrits sur l'amour et l'amitié, la politesse, la volupté, les sentiments agréables, l'esprit et le cour. Paris : édition de Thémiseul SaintHyacinthe et Anne-Thérèse de Lambert, 1736.

De Gourmont, Remy. «Essai sur l'ennui.» Promenades philosophiques. Troisième série. Reproduit à partir de la onzième édition (Paris, Mercure de France, 1925, p. 211-217).

Diderot, Denis. Les Bijoux indiscrets. In Euvres Romanesques. Edition. H. Benac. Paris : Garnier, 1965.

Dorat, Claude-Joseph. Les Sacrifices de l'amour. Paris : Gallimard, 1995.

----. Les malheurs de l'inconstance. Paris : Desjonquères, 1983. 
----. La déclamation théâtrale. In Euvres. T.4. Neuchâtel: Imprimerie de la Société Typographique, 1775.

Duclos, Charles. Les Confessions du Comte de***. Édition de Laurent Versini. Paris : Didier, 1969.

Helvétius, Claude Adrien. De l'esprit. Paris : Durand, 1758.

Laclos, Pierre Choderlos de. Les Liaisons dangereuses. Paris : GF-Flammarion, 1964.

----. Euvres Complètes. Paris : Gallimard, 1967.

La Morlière, Chevalier Charles. Angola, histoire indienne. Paris : Desjonquères, 1991.

Marmontel, Jean-François. Contes Moraux. Tome 1. Paris : Masson et Yonet, 1829.

----. Contes Moraux. Tomes 1 et 2. Paris : Dabo-Butschert, 1826.

Mauzi, Robert. L'idée du bonheur dans la littérature et la pensée françaises au XVIIIe siècle. Genève, Paris, Gex : Slatkine Reprints, 1979.

Monod, Sylvère. « Le chantre de l'ennui : Graham Greene 1978-1982 ». Études anglaises, T. XXXVI : 2-3 (1983) : 142-53.

Nordon, Didier. «L'ennui, théoricien irréfutable. » Esprit (novembre 1997) : 88-98.

Parny, Chevalier de. Poésies érotiques, Paris, 1778.

Versini, Laurent. Le roman le plus intelligent : Les Liaisons dangeureuses de Laclos. Paris : Champion Genève : Slatkine, 1998.

Villaret, Claude. Antipaméla. Paris, 1742.

Pomeau, René. Laclos : Connaissance des Lettres. Paris : Hatier, 1977.

Rosbottom, Ronald C. «Boredom and Meaning : A Reading of Laclos's Les Liaisons Dangereuses ». Forum $16: 2$ (1978) : 11-18.

Sturm, Ernest. Crébillon fils et le libertinage au dix-huitième siècle. Paris : Nizet, 1970. 


\section{NOTES}

${ }^{1}$ Crébillon Fils, Le Sylphe, Paris, Librairie des Bibliophiles, 1873, 44.

${ }^{2}$ C'est le reproche que lui fait Mme Riccoboni : «[...] Je ne suis pas surprise qu'un fils de M. de Choderlos écrive bien. L'esprit est héréditaire dans sa famille; mais je ne puis le féliciter d'employer ses talents, sa facilité, les grâces de son style, à donner aux étrangers une idée si révoltante des mœurs de sa nation et du goût de ses compatriotes. [...] On n'a pas besoin de se mettre en garde contre des caractères qui ne peuvent exister, et j'invite M. de Laclos à ne jamais orner le vice des agréments qu'il a prêtés à Mme de Merteuil » (Correspondance entre Madame Riccoboni et M. de Laclos, avril 1782, in Laclos : Euvres Complètes, édition de Maurice Allem, Paris, Gallimard, 1967, 757).

3 «Libertinage : C'est l'habitude de céder à l'instinct qui nous porte aux plaisirs des sens » (Encyclopédie ou Dictionnaire raisonné des sciences, des arts et des métiers, par une société de gens de lettres, T. IX, 476).

${ }^{4}$ Il y a une affirmation semblable dans Les Égarements du cour et de l'esprit de Crébillon Fils où Madame de Senanges déclare : «On est amoureux quelques temps. Les yeux s'ouvrent à la fin, on voit ce qu'on a pris, on s'ennuie de l'avoir, on en rougit, et l'on quitte » (145).

${ }^{5}$ Voir Marc André Bernier, Libertinage et figures du savoir: Rhétorique et roman libertin dans la France des Lumières (1734-1751), Québec, Presses de l'Université Laval, 2001, 184.

${ }^{6}$ Pour une étude sur le désir dans l'œuvre de Diderot, voir J. E. Fowler, Voicing Desire : Family and sexuality in Diderot's Narrative, Oxford, 2000.

${ }^{7}$ Dans Fanny Hill, la fille de joie, ce sont les parties intimes du corps qui revivifient les sentiments « anéantis » de l'héroïne. Fanny se trouve dans les bras de son amant «sans aucun sentiment, excepté dans ces parties favorites de la nature où nos âmes, notre vie et toutes nos sensations étaient alors entièrement concentrées (Fougeret de Monbron, Fanny Hill, la fille de joie, 64). À proprement parler, l'idée du sentiment n'existe guère en dehors du corps.

${ }^{8}$ La Morlière expose la conception de l'amour des libertins par la bouche d'Almaïr: «Nous dissertions sérieusement sur l'amour, Madame, répondit Almair, et sur tous les inconvénients où on s'expose en s'y livrant, et nous convenions, le prince et moi, que ce n'est qu'en le fuyant, ou en le traitant cavalièrement qu'on peut se soustraire à sa tyrannie (Angola, 149).

${ }^{9}$ Dans cet article, Nordon définit la difficulté comme une « arme contre l'ennui ».

${ }^{10}$ Voir Philippe Laroch, Petits-maîtres et roués : évolution de la notion de libertinage dans le roman français du XVIII siècle. Québec, PU Laval, 1979, 337.

${ }^{11}$ Il faut souligner que la notion de bonheur reste passablement floue au XVIIIe siècle. Pour une étude du bonheur au XVIIIe siècle, nous invitons le lecteur à lire l'ouvrage de Robert Mauzi, L'idée du bonheur dans la littérature et la pensée françaises au XVIIIe siècle, Genève, Paris, Gex, Slatkine Reprints, 1979.

12 Sylvère Monod a raison d'affirmer que : « la volupté devrait être un moyen d'échapper à l'ennui, mais elle inspire vite le dégoût, l'horreur, ou l'ennui à son tour. » («Le chantre de l'ennui : Graham Greene 1978-1982 », Études anglaises, T. XXXVI : 2-3 (1983), 142).

${ }^{13}$ L'expression vient de Laurent Versini dans Laclos et la tradition, essai sur les ressources et la technique des Liaisons dangereuses, 59.

${ }^{14}$ Peut-être serait-il plus légitime de parler des « riens de l'amour. » Cette métaphore utilisée par Dorat témoigne de la vacuité de l'amour et sert à minimiser son importance :

Tous ces riens de l'amour, savez vous les bien dire?

Pour le représenter, avez-vous ses appas?

Il enlaidit toujours ceux qu'il n'embellit pas.

Charmant, vous n'avez rien et vous devez tout craindre, si vous ignorez l'art d'exprimer et de peindre,

de produire au dehors ces orages du coeur,

ces mouvemens secrets, ces instans de fureur,

ces rapides retours, cette brûlante ivresse,

les transports de l'amour et sa délicatesse (Claude-Joseph Dorat, La déclamation théâtrale, 73). 